\title{
Multistage communication with and without verifiable types
}

\author{
Frédéric Koessler * Françoise Forges ${ }^{\dagger}$
}

September 22, 2006

\begin{abstract}
We survey the main results on strategic information transmission, which is often referred to as "persuasion" when types are verifiable and as "cheap talk" when they are not. In the simplest "cheap talk" model, an informed player sends a single message to a receiver who makes a decision. The players' utilities depend on the sender's information and the receiver's decision, but not on the sender's message. Furthermore, the messages that are available to the sender do not depend on his true information. As is well-known, such a unilateral "cheap talk" can affect the sender's decision at equilibrium.

In a more general model, both players can exchange simultaneous costless messages during several stages before the final decision. The utility functions are unchanged. Multistage conversation allows the players to reach more equilibrium outcomes, which possibly Pareto dominate the original ones. More precisely, the set of equilibrium outcomes of long cheap talk games is fully characterized; it increases with the number of communication stages and can become even larger if no deadline is imposed.

Concentrating on cheap talk is not appropriate if the informed player can influence the decision maker by producing unfalsifiable documents. In order to capture this possibility formally, one assumes that the informed player's set of messages depends on his private information. The literature has mostly dealt with unilateral persuasion. But multistage, bilateral communication enables the players to reach more equilibrium outcomes in the case of verifiable types as in the case of unverifiable ones. Equilibria of long persuasion games are fully characterized when information can be certified at any precision level.
\end{abstract}

Keywords: Cheap talk; certification; incomplete information; information transmission; jointly controlled lotteries; verifiable types.

JEL Classification: C72, D82.

\section{Introduction}

Models of strategic information transmission fall into two categories, according to whether the agents' information is verifiable or not. The term "persuasion game" is often associated with the first framework, while "cheap talk" typically refers to the second one.

Crawford and Sobel (1982) studied the effects of cheap talk in a particularly tractable game. An informed expert sends a message to a decision maker. The same messages are available to the expert, whatever his information. Both agents' utility depends on the sender's

\footnotetext{
${ }^{*}$ THEMA, Université de Cergy-Pontoise, 33 Boulevard du Port, F-95011 Cergy-Pontoise, France, E-mail: Frederic.Koessler@u-cergy.fr URL: www.u-cergy.fr/rech/pages/koessler/

${ }^{\dagger}$ CEREMADE, Université de Paris-Dauphine, Place du Maréchal de Lattre de Tassigny, F-75775 Paris Cedex 16, France, E-mail: Francoise.Forges@dauphine.fr
} 
information and on the receiver's action, but not on the message, which is thus costless. Crawford and Sobel (1982) assume that the information states and the actions belong to a real interval and that the utility functions satisfy specific properties. They characterize the Nash equilibria of the game and show in particular that even in the absence of signalling cost, information transmission can matter at equilibrium. We describe this result in section 2.3.

Before that, in section 2.1, we consider the class of all sender-receiver games in which the set of information states and the set of actions are both finite, without imposing any restriction on the utility functions. As in the previous model, the game involves a single stage of information transmission, from the informed agent to the decision maker, and the set of costless messages available to the sender does not depend on his private information. Section 2.1 describes a geometric characterization of all Nash equilibria of the game of information transmission. This result can be deduced from a characterization of Nash equilibria in repeated games with incomplete information (see Aumann, Maschler, and Stearns, 1968, Hart, 1985, and Aumann and Hart, 2003). Examples illustrate different possible kinds of Nash equilibria: nonrevealing, fully revealing, and partially revealing. Section 2.2 briefly states some results for the particular class of "monotonic" games.

In standard cheap talk, the informed player can use the same messages, whatever his information, which is thus unverifiable. As pointed out, e.g., by Green and Laffont (1986), in some applications, the type of an agent determines the signals he can send. For instance, if the sender privately knows his endowment, the receiver might require a concrete deposit instead of cheap talk. In this case, the sender will not be able to pretend that he is richer than he really is. "Certificates", which depend on the sender's information, can thus sometimes be added to cheap talk messages. By making imitation hard or even impossible, such certificates may help the informed player to reveal his information to a rational receiver.

The simplest version of a persuasion game involves an expert and a decision maker as in section 2. The only difference is that the set of messages that are available to the sender now depends on his information. In section 3, we first characterize Nash equilibria in the case of finite sets of types and actions, as in Forges and Koessler (2006). Standard refinements of Nash equilibrium, as the perfect Bayesian equilibrium, which have no effects in cheap talk games, can be quite powerful in persuasion games. This is illustrated in sections 3.2 and 3.3. Following Milgrom (1981), several papers establish the existence, or even the uniqueness, of a fully revealing equilibrium in different classes of persuasion games, in particular in a version of Crawford and Sobel's model with verifiable types.

In the models surveyed above, only the expert can talk, in a single phase of information transmission. However, in their seminal study of two-person repeated games with lack of information on one side, Aumann, Maschler, and Stearns (1968) show on examples that new equilibria can be achieved if two or three stages of communication are feasible to the players. They emphasize the crucial role of the uniformed player in this multistage communication: the stages of pure information transmission, which are handled by the informed agent, alternate with stages of compromise, in which both agents agree on the way to interact in the future. 
Hart (1985) fully characterized Nash equilibria in the repeated games introduced by Aumann, Maschler, and Stearns. In that paper, the informed player not only sends costless messages but also makes decisions which influence both players' utilities. The equilibria characterized by Hart reflect strategic information transmission, but also complex cooperation behavior (as in the "Folk theorem").

However, Hart's characterization can be easily adapted to a variant of sender-receiver games in which both agents talk cheaply, as long as they wish, before the decision maker selects an action. By adapting Hart (1985)'s results to that framework, one can show that the set of equilibria increases with the number of communication stages. Even more, some equilibria cannot be achieved, unless no deadline is imposed to the communication (see Forges, 1984, 1990, 1994). In section 4.1, we describe a geometric characterization of all Nash equilibria which can be achieved with finitely many stages of bilateral cheap talk, when the sets of types and actions are finite. This result is based on Hart (1985) and Aumann and Hart (2003), who use the techniques of infinitely repeated games to analyze "long cheap talk". ${ }^{1}$

The previous results hold under the assumption that the sets of types and actions are finite. What about Crawford and Sobel's famous model? Krishna and Morgan (2004) show that, if both the expert and the decision maker can talk for a few stages, Crawford and Sobel's partitional equilibria can be (ex ante) Pareto improved. We present that result in section 4.2 .

The review above shows that the effects of cheap talk, persuasion and long cheap talk have deserved much attention. There remains to investigate "long persuasion", namely multistage bilateral communication when the expert's information is verifiable. In section 5, an example, from Forges and Koessler (2006), shows that the informed player can then profitably delay the certification of information. We describe a characterization of all equilibria which can be achieved with finitely many stages of bilateral communication, assuming that the expert can certify his information at any precision level.

\section{Unverifiable Information: Cheap Talk Games}

In this section, we deal with the standard model of information transmission, in which an informed expert sends a single costless message to a decision maker. We characterize Nash equilibria, first in the case of finitely many types and actions, then in monotonic games and in Crawford and Sobel's model.

\footnotetext{
${ }^{1}$ Infinitely repeated games provide insights on strategic communication, but are technically very different from long cheap talk games. For instance, a nonrevealing equilibrium always exists in Aumann and Hart (2003), even if the informed player has to make a payoff relevant decision at the end. However, proving the existence of an equilibrium in Hart (1985) is extremely hard (see Renault, 2000, Simon, Spiez, and Torunczyk, 1995, and Sorin, 1983).
} 


\subsection{A geometric characterization of Nash equilibria}

Let $K$ be the finite set of possible states of information, or types, of the expert, player 1 . We assume for expository purposes that there are only two possible types: $K=\left\{k_{1}, k_{2}\right\}=\{1,2\}$. The decision maker, player 2 , has no private information; he must choose an action $j$ in a finite set $J$. Let $\operatorname{Pr}\left(k_{1}\right)=p$ and $\operatorname{Pr}\left(k_{2}\right)=1-p$. Given a state $k$ and an action $j$, the utilities of player 1 and player 2 are denoted as $A^{k}(j)$ and $B^{k}(j)$ respectively. The corresponding decision problem, or game without communication, is denoted as $\Gamma(p)$.

Let $y \in \Delta(J)$ be a mixed strategy of the decision maker in $\Gamma(p)$. The corresponding expected payoffs are $A^{k}(y)=\sum_{j \in J} y(j) A^{k}(j)$ and $B^{k}(y)=\sum_{j \in J} y(j) B^{k}(j)$. The equilibria of $\Gamma(p)$, which will be referred to as nonrevealing equilibria, are simply the optimal mixed strategies of the decision maker, given his belief $p$ over the expert's type:

$$
Y(p) \equiv \arg \max _{y \in \Delta(J)}\left[p B^{1}(y)+(1-p) B^{2}(y)\right]
$$

The set of all equilibrium payoffs of $\Gamma(p)$ can be described as

$$
\mathcal{E}(p) \equiv\left\{(a, \beta) \in \mathbb{R}^{2} \times \mathbb{R}: \exists y \in Y(p), a=A(y), \beta=p B^{1}(y)+(1-p) B^{2}(y)\right\}
$$

The unilateral cheap talk game is constructed by adding a single phase of communication, from the expert to the decision maker, between the information phase (in which the expert learns his type) and the decision phase (in which the decision maker chooses an action). In the communication phase, the expert sends a message $m \in M$, with $3 \leq|M|<\infty$. The unilateral communication game is denoted as $\Gamma_{S}^{0}(p) .^{2}$ In this game, a strategy of the expert is a mapping $\sigma: K \rightarrow \Delta(M)$, which associates a probability distribution over the set of messages $M$ to each possible type. A strategy of the decision maker is a mapping $\tau: M \rightarrow \Delta(J)$, which associates a probability distribution over the set of actions $J$ to every possible message. Let us denote as $\mathcal{E}_{S}^{0}(p)$ the set of all Nash equilibrium payoffs of the unilateral communication game $\Gamma_{S}^{0}(p)$.

An equilibrium of $\Gamma_{S}^{0}(p)$ is fully revealing (FRE) if player 1 sends a different message for each of his possible types. It is nonrevealing (NRE) if player 2's strategy is independent of the messages that are sent at equilibrium. It is partially revealing (PRE) if it does not fall in one of the previous two categories.

It is easily checked that a cheap talk game always has a NRE in which the expert sends the same message whatever his type and the decision maker uses the same optimal strategy in the game without communication whatever the expert's message. In other words, $\mathcal{E}(p) \subseteq \mathcal{E}_{S}^{0}(p)$. We will describe a geometric characterization of $\mathcal{E}_{S}^{0}(p)$, adapted from Hart (1985) (see also Forges, 1994).

Let us denote as $\mathcal{E}^{+}(p)$ the set of modified equilibrium payoffs of the game without com-

\footnotetext{
${ }^{2}$ The upper index " 0 " indicates that information is not certifiable. The lower index "S" stands to recall that the game is a signalling game, by contrast with bilateral communication.
} 
munication $\Gamma(p)$, in which the expert's payoffs are extended when $p=0$ or $p=1$, by allowing a zero probability type to make a (virtual) gain that exceeds the equilibrium payoff. More precisely, $\mathcal{E}^{+}(p)$ is the set of payoffs $(a, \beta) \in \mathbb{R}^{2} \times \mathbb{R}$ such that there exists an equilibrium $y \in Y(p)$ of $\Gamma(p)$ verifying the following conditions:

(i) $a^{k} \geq A^{k}(y)$, for every $k \in K$;

(ii) $a^{1}=A^{1}(y)$ if $p \neq 0$ and $a^{2}=A^{2}(y)$ if $p \neq 1$;

(iii) $\beta=p B^{1}(y)+(1-p) B^{2}(y)$.

In particular, $\mathcal{E}^{+}(p)=\mathcal{E}(p)$ if $\left.p \in\right] 0,1[$. The graph of the modified equilibrium payoff correspondence is denoted as

$$
\operatorname{gr} \mathcal{E}^{+} \equiv\left\{(a, \beta, p) \in \mathbb{R}^{2} \times \mathbb{R} \times[0,1]:(a, \beta) \in \mathcal{E}^{+}(p)\right\}
$$

Theorem 1 (Characterization of $\mathcal{E}_{S}^{0}(p)$ ) Let $\left.p \in\right] 0,1[. \quad A$ payoff $(a, \beta)$ is an equilibrium payoff of the unilateral communication game $\Gamma_{S}^{0}(p)$ if and only if $(a, \beta, p)$ belongs to $\operatorname{conv}_{a}\left(\operatorname{gr} \mathcal{E}^{+}\right)$, the set of all points that can be obtained by convexifying the set $\operatorname{gr} \mathcal{E}^{+}$in $(\beta, p)$ while keeping constant the expert payoff, a:

$$
\mathcal{E}_{S}^{0}(p)=\left\{(a, \beta) \in \mathbb{R}^{2} \times \mathbb{R}:(a, \beta, p) \in \operatorname{conv}_{a}\left(\operatorname{gr} \mathcal{E}^{+}\right)\right\}
$$

See Hart (1985), Forges (1994) and Aumann and Hart (2003) for a proof. The result remains true for an arbitrary number $\kappa$ of expert's types, provided that $|M| \geq \kappa+1$. Let us illustrate the characterization on examples.

Example 1 (No revelation of information) The game without communication and its equilibria are given by figure $1 .^{3}$ The graph of the modified equilibrium payoffs, gr $\mathcal{E}^{+}$, is represented in the expert's payoff space on figure 2. The absence of any intersection point indicates that no new point can be obtained by convexifying the graph when the expert's vector payoff, $a=\left(a^{1}, a^{2}\right)$, is fixed. Hence, whatever the prior probability $\left.p \in\right] 0,1[$, the set of equilibrium payoffs is the same, whether communication is allowed or not: $\mathcal{E}(p)=\mathcal{E}_{S}^{0}(p)$.

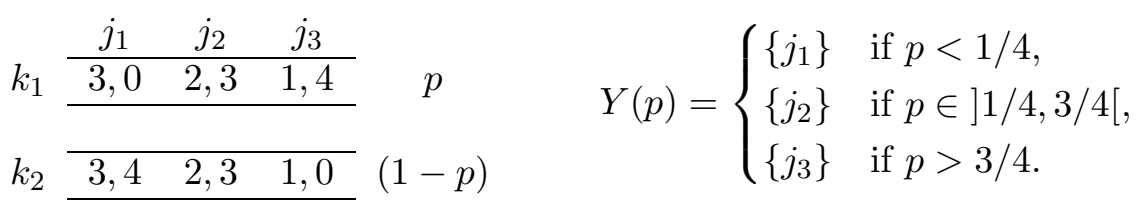

Figure 1: Game without communication and optimal actions in example 1.

\footnotetext{
${ }^{3}$ We do not indicate the optimal mixed actions for values of $p$ at the border of the intervals; obviously, $Y(p)=\Delta\left(\left\{j_{1}, j_{2}\right\}\right)$ when $p=1 / 4$, and $Y(p)=\Delta\left(\left\{j_{2}, j_{3}\right\}\right)$ when $p=3 / 4$.
} 


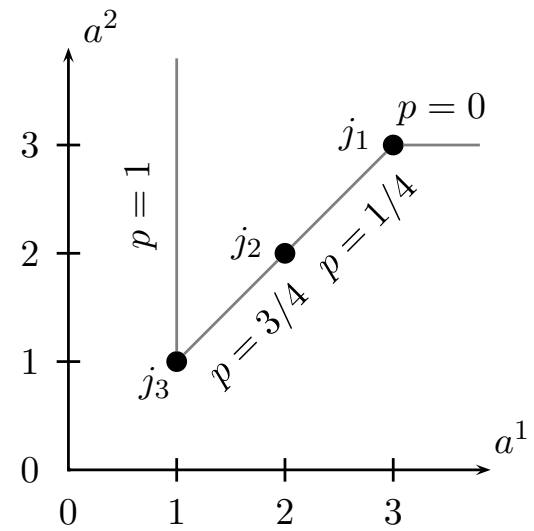

Figure 2: Expert's modified equilibrium payoffs in example 1.

Example 2 (Credible revelation of information) Consider the game without communication of figure 2. The graph of the modified equilibrium payoffs is represented on figure 4 . The only point of intersection is $(1,3)$, at $p=0$ and at $p=1$. Hence, by convexifying the graph in $p$ while fixing $(1,3)$, one gets $(1,3)$ as a fully revealing equilibrium payoff at every $p \in] 0,1[$.

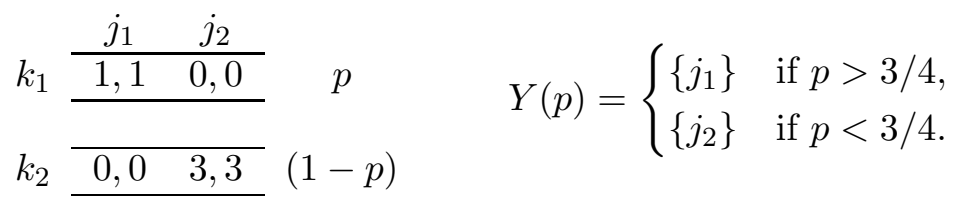

Figure 3: Game without communication and optimal actions in example 2.

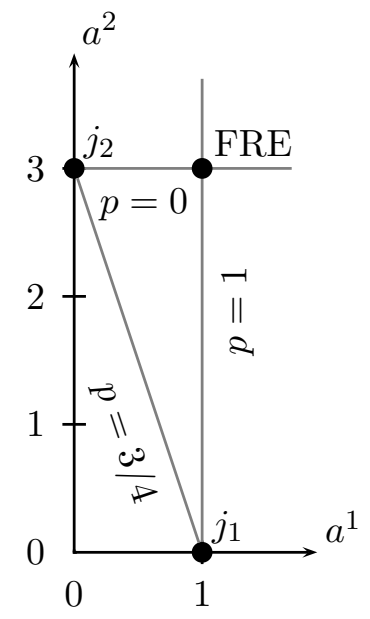

Figure 4: Expert's modified equilibrium payoffs in example 2.

Example 3 (Partial revelation of information) The basic game and the optimal actions of the decision maker, as a function of $p$, are described by figure 5 . The graph of 
the modified equilibrium payoffs is depicted in figure 6 . For $p \notin] 3 / 10,4 / 5[$ all equilibria of the game with communication are nonrevealing, while for $p \in] 3 / 10,4 / 5[$, there exists a PRE yielding the vector payoff $(11 / 4,23 / 8)$ to the expert.

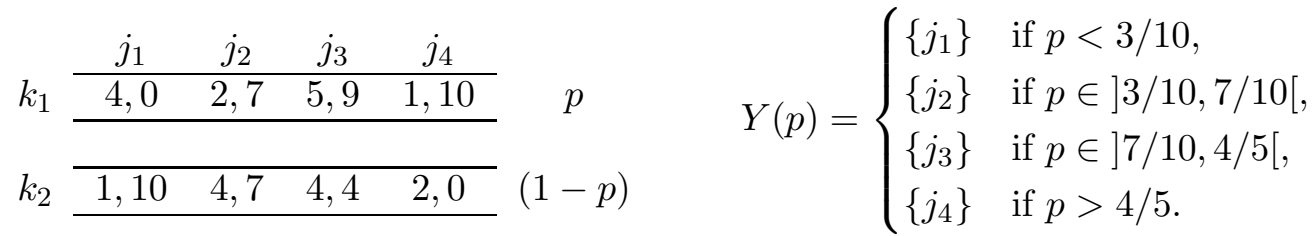

Figure 5: Game without communication and optimal actions in example 3.

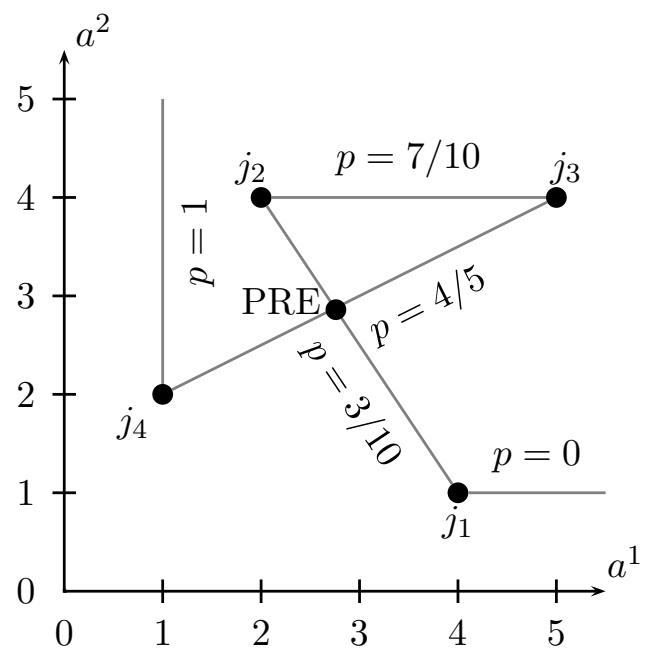

Figure 6: Expert's modified equilibrium payoffs in example 3.

\subsection{Monotonic games}

The preferences of the expert are monotonic if the decision maker's actions can be ordered in such a way that

$$
A^{k}(j)>A^{k}\left(j^{\prime}\right) \Leftrightarrow j>j^{\prime}, \quad \forall k \in K
$$

Monotonic games have been extensively studied in the context of verifiable information (see section 3$)$.

If the expert has two possible types and the optimal action of the decision maker is monotonic in his belief $p$ on $k_{1}$ (as in example 1), then the geometric characterization of theorem 1 shows that the unique equilibrium of the communication game is nonrevealing, whatever the prior $p$, since the graph of the expert's modified equilibrium payoffs has no intersection point. More generally, in a monotonic communication game such that $|K| \geq 2$, it is easy to show that every Nash equilibrium in which the decision maker plays a pure strategy is nonrevealing. ${ }^{4}$ This conclusion also applies in monotonic games in which $J$ is a

\footnotetext{
${ }^{4}$ This result can be proved along the same lines as Watson (1996, theorem 1). Notice that partially and
} 
real interval (instead of being finite) and $B^{k}(\cdot)$ is strictly concave over $J$ for every $k$, since the decision maker then plays a pure strategy (see, e.g., Grossman and Hart, 1980, Grossman, 1981, Milgrom, 1981, Milgrom and Roberts, 1986).

\subsection{Partitional equilibria: Crawford and Sobel's model}

Crawford and Sobel (1982) is one of the first articles on costless strategic information transmission (see also Green and Stokey, 1980). We concentrate here on the simplest version of the model, known as "uniform-quadratic". The expert has a continuum of possible types in $K=[0,1]$; similarly, his set of messages is $M=[0,1]$. The set of actions of the decision maker is $J=[0,1]$. The prior probability distribution over $K$ is uniform. The utility functions of the expert and the decision maker have the following form:

$$
\begin{aligned}
& A^{k}(j)=-[j-(k+b)]^{2}, \quad b>0, \\
& B^{k}(j)=-[j-k]^{2} .
\end{aligned}
$$

When $k$ increases, both players prefer that the action increases but the expert's ideal action, $j_{1}^{*}(k)=\arg \max _{j} A^{k}(j)=k+b$, is always strictly greater than the decision maker's one, $j_{2}^{*}(k)=\arg \max _{j} B^{k}(j)=k$. The parameter $b$ can thus be interpreted as the "bias of the expert".

Crawford and Sobel consider a single stage of information transmission, from the expert to the decision maker. They characterize the equilibria of the game by showing that they are all "partitional". More precisely, the equilibrium strategy of the expert $\sigma: K \rightarrow M$ consists of sending $n$ different messages, for some integer $n$ : $\sigma(k)=m_{1}$ if $k \in\left[0, x_{1}\left[, \ldots, \sigma(k)=m_{l}\right.\right.$ if $k \in\left[x_{l-1}, x_{l}\left[, \ldots, \sigma(k)=m_{n}\right.\right.$ if $k \in\left[x_{n-1}, 1\right]$, with $0<x_{1}<\cdots<x_{n-1}<x_{n}=1, m_{l} \neq m_{l^{\prime}}$ $\forall l \neq l^{\prime}$.

By expressing the decision maker's best reply conditions and going back inductively to the expert's equilibrium conditions, one gets

$$
x_{l}=l / n-2 l b(n-l), \quad l=1, \ldots, n .
$$

The fact that the $x_{l}$ 's must remain positive yields the following condition for the existence of a partitional equilibrium with $n$ different messages, also called $n$-separating equilibrium:

$$
x_{1}=1 / n-2 b(n-1)>0 \quad \Leftrightarrow \quad b<\frac{1}{2 n(n-1)} .
$$

Given a bias $b$, the greatest $n$ for which there exists an $n$-separating equilibrium is thus the greatest $n$ such that $2 n(n-1) b<1$ (which is equal to 2 if $b=1 / 4$ and goes to $+\infty$ as $b \rightarrow 0)$.

It is difficult to compare the different equilibria in terms of interim efficiency since the fully revealing equilibria may exist in monotonic games in which the decision maker plays in mixed strategies. 
preferred equilibrium of the expert depends in general on his type. However, the equilibria can be compared ex ante: both players always prefer the equilibrium in which the largest number of different messages are sent. In particular, cheap talk ex ante Pareto improves upon the equilibria of the game without communication.

Crawford and Sobel (1982) extend their approach by assuming, basically, that:

(A) The prior probability distribution over types has a density.

(B) The utility functions $A^{k}(\cdot)$ and $B^{k}(\cdot)$ are concave in $j$ for every $k \in K$, and $j_{1}^{*}(k)=$ $\arg \max _{j \in J} A^{k}(j), j_{2}^{*}(k)=\arg \max _{j \in J} B^{k}(j)$ are unique, continuous and increasing in $k$.

(C) $j_{1}^{*}(k) \neq j_{2}^{*}(k)$ for every $k \in K$, so $D(k)=j_{2}^{*}(k)-j_{1}^{*}(k)$ has always the same sign. ${ }^{5}$

In the more general model, they show that all equilibria are $n$-separating, and that such equilibria exist for increasing values of $n$ when the players' preferences become more similar to each other. However, in general, equilibria cannot be compared in terms of ex ante efficiency.

\section{Verifiable Information: Persuasion Games}

In the previous section, we have assumed that the communicating abilities of a player did not depend on his knowledge. We relax this assumption. The messages that can possibly be sent by the informed player now depend on his type, i.e., on his private information. This information is thus (partially) verifiable or certifiable. The corresponding unilateral communication game is traditionally referred to as a persuasion game. In this case, it may be hard or even impossible for a player's type to imitate another. Hence the set of equilibrium outcomes is in general quite different from the one that is achieved with pure cheap talk.

We state an analog of theorem 1, namely a geometric characterization of Nash equilibria in persuasion games and illustrate it on an example. We then study the effect of some standard equilibrium refinements (subgame perfect equilibrium and perfect Bayesian equilibrium) which have no bite on cheap talk games but prove useful in persuasion games. We finally consider certification in a framework that extends Crawford and Sobel (1982)'s one.

\subsection{A geometric characterization of Nash equilibria}

The timing of the unilateral persuasion game, denoted as $\Gamma_{S}(p)$, is the same as in the unilateral cheap talk game $\Gamma_{S}^{0}(p)$, except that the set of messages that are available to the expert depends on $k$, and is denoted as $M(k), k \in K$. Let $M^{1}=M\left(k_{1}\right) \cup M\left(k_{2}\right)$ be the set of all possible messages for the expert. We assume that the expert can certify any of his types (i.e., for every $k$ there exists $c^{k} \in M^{1}$ such that $\left.M^{-1}\left(c^{k}\right)=\{k\}\right)$ and that there are at least three cheap talk messages, which do not certify any information (i.e., $\left|M\left(k_{1}\right) \cap M\left(k_{2}\right)\right| \geq 3$ ).

\footnotetext{
${ }^{5}$ In Melumad and Shibano (1991), Gordon (2006) and sub-section 3.3, this assumption is relaxed, i.e., $D(k)$ can change sign.
} 
A strategy of the expert in the persuasion game is a mapping $\sigma: K \rightarrow \Delta\left(M^{1}\right)$ such that $\operatorname{supp} \sigma(k) \subseteq M(k)$. A strategy of the decision maker is a mapping $\tau: M^{1} \rightarrow \Delta(J)$. We denote as $\mathcal{E}_{S}(p)$ the set of all Nash equilibrium payoffs of the unilateral persuasion game $\Gamma_{S}(p)$.

When we characterized the Nash equilibria of the unilateral cheap talk game, we considered the set $\mathcal{E}^{+}(p)$ of modified equilibrium payoffs of the game without communication $\Gamma(p)$. Here, we consider the larger set $\mathcal{E}^{++}(p)$ of extended equilibrium payoffs of $\Gamma(p)$, for which there exists $y \in Y(p)$ such that properties (ii) and (iii) on page 5 (but not property (i)) are satisfied. $\mathcal{E}^{++}(p)$ is thus the set of equilibrium payoffs of the game without communication, except that, at a type of zero probability, the expert can get any payoff (above but also below his equilibrium payoff). We denote as gr $\mathcal{E}^{++} \equiv\left\{(a, \beta, p) \in \mathbb{R}^{2} \times \mathbb{R} \times[0,1]:(a, \beta) \in \mathcal{E}^{++}(p)\right\}$ the graph of the correspondence of extended equilibrium payoffs and as

$$
\operatorname{INTIR} \equiv\left\{(a, \beta, p) \in \mathbb{R}^{2} \times \mathbb{R} \times[0,1]: \exists \bar{y} \in \Delta(J), a^{k} \geq A^{k}(\bar{y}) \forall k \in K\right\},
$$

the set of all points $(a, \beta, p)$ such that $a$ is interim individually rational for the expert.

Theorem 2 (Characterization of $\left.\mathcal{E}_{S}(p)\right)$ Let $\left.p \in\right] 0,1[$. A payoff $(a, \beta)$ is an equilibrium payoff of the unilateral persuasion game $\Gamma_{S}(p)$ if and only if $(a, \beta, p)$ belongs to $\operatorname{conv}_{a}\left(\mathrm{gr}^{++}\right) \cap$ INTIR, the set of all points obtained by convexifying the set gr $\mathcal{E}^{++}$in $(\beta, p)$ while keeping constant and individually rational the expert's payoff, a:

$$
\mathcal{E}_{S}(p)=\left\{(a, \beta) \in \mathbb{R}^{2} \times \mathbb{R}:(a, \beta, p) \in \operatorname{conv}_{a}\left(\operatorname{gr} \mathcal{E}^{++}\right) \cap \operatorname{INTIR}\right\}
$$

See Forges and Koessler (2006) for a proof. The theorem remains true for an arbitrary number of types of the expert provided that the latter can certify any subset of types $L \subseteq K$.

Let us first illustrate the previous characterization on example 1 (figure 1 on page 5 ). We have seen that revelation of information through cheap talk was not credible. One checks immediately that, if each type $k$ can send a certificate, $m=c^{k}$, that is only available to type $k$, the game has a fully revealing equilibrium. The next example taken from Forges and Koessler (2006), exhibits other kinds of equilibria with information certification, some of them with partial certification. We will come back to it to illustrate the impact of multistage, bilateral communication in section 5 .

Example 4 Consider the game without communication of figure 7, with $\operatorname{Pr}\left[k_{1}\right]=1 / 2$.

Let $c^{k}$ be a certificate for type $k$, and $\bar{m} \in M\left(k_{1}\right) \cap M\left(k_{2}\right)$ be a cheap talk message. There exist a FRE and a NRE, with respective vector payoffs $(2,1)$ and $(0,0)$ for the expert. There are also two partially revealing equilibria; one of them, denoted as PRE1, gives the expert a higher payoff than both pure strategy equilibria. At PRE1, player 1 certifies his information (namely, he sends the message $c^{1}$ ) with probability $1 / 3$ and remains silent, i.e., sends the message $\bar{m}$, with probability $2 / 3$ if his type is $k_{1}$; he always remains silent, i.e., 


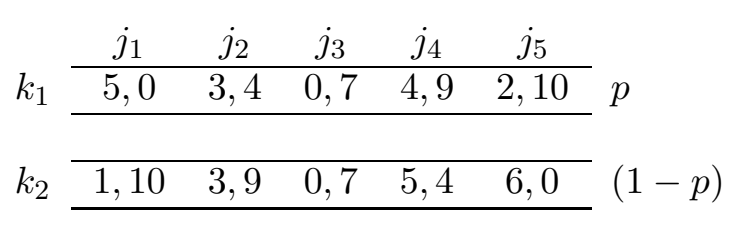

$$
Y(p)= \begin{cases}\left\{j_{1}\right\} & \text { if } p<1 / 5, \\ \left\{j_{2}\right\} & \text { if } p \in] 1 / 5,2 / 5[ \\ \left\{j_{3}\right\} & \text { if } p \in] 2 / 5,3 / 5[ \\ \left\{j_{4}\right\} & \text { if } p \in] 3 / 5,4 / 5[ \\ \left\{j_{5}\right\} & \text { if } p>4 / 5\end{cases}
$$

Figure 7: Game without communication and optimal actions in example 4.

sends the message $\bar{m}$ if his type is $k_{2}$. The posterior beliefs of player 2 are then $\operatorname{Pr}\left(k_{1} \mid \bar{m}\right)=$ $\frac{\operatorname{Pr}\left(\bar{m} \mid k_{1}\right) \operatorname{Pr}\left(k_{1}\right)}{\operatorname{Pr}(\bar{m})}=2 / 5$ and $\operatorname{Pr}\left(k_{1} \mid c^{1}\right)=1$, so that he plays action $j_{5}$ when he receives message $c^{1}$, and he is indifferent between $j_{2}$ and $j_{3}$ when he receives message $\bar{m}$. If he chooses $j_{2}$ with probability $2 / 3$ and $j_{3}$ with probability $1 / 3$ on $\bar{m}$, and chooses $j_{1}$ on the out of equilibrium message $c^{2}$, player 1 cannot gain in deviating and his vector payoff is equal to $(2,2)$. The second partially revealing equilibrium, which we denote as PRE2, is symmetric to PRE1: player 1 always sends message $\bar{m}$ on $k_{1}$, and sends message $c^{2}$ with probability $1 / 3$ and message $\bar{m}$ with probability $2 / 3$ on $k_{2}$, and player 2 chooses $j_{1}$ on $c^{2}, \frac{4}{5} j_{3}+\frac{1}{5} j_{4}$ on $\bar{m}$ and $j_{3}$ on the out of equilibrium message $c^{1}$. At PRE2, player 1's expected payoff is $(4 / 5,1)$. If the prior probability of the first type is $\operatorname{Pr}\left[k_{1}\right]=1 / 2$, there is no other Nash equilibrium.

Let us make the connection with the geometric characterization stated above. The graph of the correspondence of modified equilibrium payoffs, gr $\mathcal{E}^{+}$, is represented in the expert's payoff space by solid lines on figure 8 on the following page. According to theorem 1 , the absence of any intersection point means that all equilibria of the cheap talk game are nonrevealing. The graph of the correspondence of extended equilibrium payoffs, gr $\mathcal{E}^{++}$, is represented on the same figure by the solid and dashed lines. Since all the points at the North-East of $(0,0)$ are interim individually rational, the convexification of $\mathrm{gr}^{++}$, keeping the expert payoff constant and individually rational, generates three new points at $p=1 / 2$ : FRE, PRE1 and PRE2, which are exactly the three Nash equilibrium payoffs identified previously, in addition to the NRE. Observe that PRE3, for instance, is not an equilibrium payoff at $p=1 / 2$ since $1 / 2 \notin[3 / 5,1]$.

The standard equilibrium refinements are useless in cheap talk games (see for instance Kreps and Sobel, 1994 and Blume, 1994). This is no longer the case in persuasion games in which some Nash equilibria clearly depend on non-credible threats. For instance, in example 4, the nonrevealing equilibrium (NRE) and the second partially revealing equilibrium (PRE2) are not subgame perfect. Indeed, in the subgame following certificate $c^{1}$, the only optimal decision is $j_{5}$, and in the subgame following $c^{2}$, the only optimal decision is $j_{1}$.

In persuasion games, the geometric characterization of subgame perfect equilibrium (SPE) payoffs, or of perfect Bayesian equilibrium (PBE) payoffs, is obtained by strengthening the interim individual rationality condition of the expert. In an SPE, when the expert certifies out of equilibrium that his type is $k$, the decision maker must choose an action that is optimal 


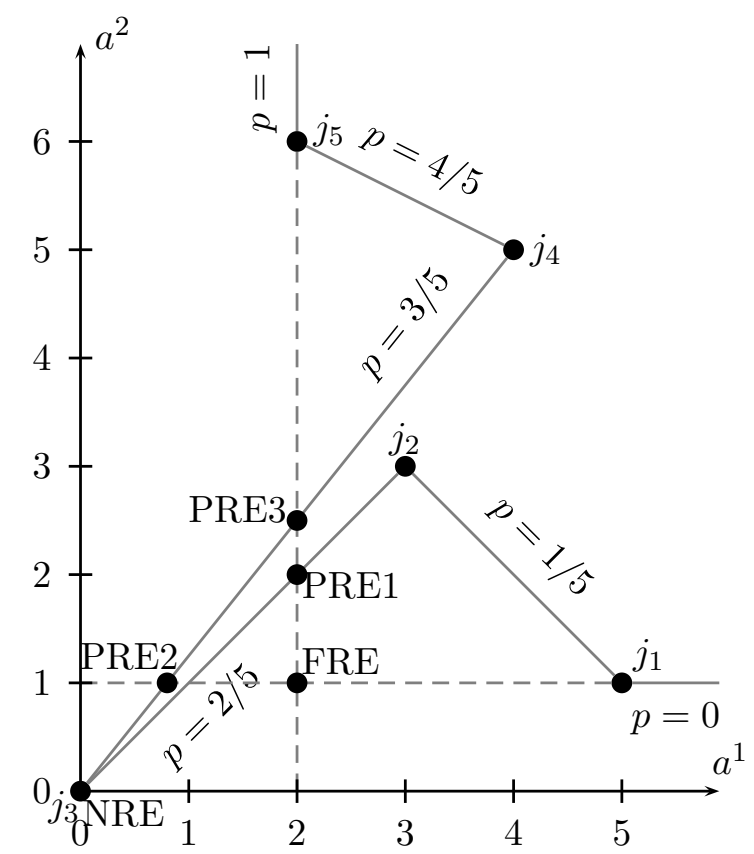

Figure 8: Modified (solid lines) and extended (solid and dashed lines) equilibrium payoffs of the expert in example 4.

when the expert's type is $k$. In a $\mathrm{PBE}$, one must require in addition that given an out of equilibrium cheap talk message, the decision maker's action be optimal for some beliefs over $K$. Formally, in the geometric characterization of theorem 2, the expert's payoff, $a=\left(a^{1}, a^{2}\right)$, must satisfy the following additional conditions:

$$
\begin{aligned}
& \exists \bar{y}_{1} \in Y(1) \text { such that } a^{1} \geq A^{1}\left(\bar{y}_{1}\right) \\
& \exists \bar{y}_{2} \in Y(0) \text { such that } a^{2} \geq A^{2}\left(\bar{y}_{2}\right),
\end{aligned}
$$

at a SPE, together with

$$
\exists p \in \Delta(K), \bar{y} \in Y(p) \text { such that } a^{k} \geq A^{k}(\bar{y}) \forall k \in K,
$$

at a PBE. These requirements are easily adapted when there are more than two types (see Forges and Koessler, 2006). In the sequel of this section, the equilibrium concept is PBE, unless explicitly mentioned.

\subsection{Monotonic games and skepticism}

Let us go back to monotonic games, with an arbitrary set of types $K$. We have seen that, in these games, cheap talk did not enable the expert to credibly reveal his information (see section 2.2). Let us assume now that each type can be fully certified: for every $k \in K$, there exists $m \in M(k)$ such that $M^{-1}(m)=\{k\}$. A skeptical strategy for the decision maker consists of choosing the minimal action among his best replies, given the types that are 
compatible with the received message. By relying on such strategies, one can show that any monotonic persuasion game has a fully revealing equilibrium. Under further assumptions, for instance if $J$ is a real interval and $B^{k}(j)$ is strictly concave in $j$ for every $k$, this FRE is unique (see Milgrom, 1981, Grossman, 1981, Milgrom and Roberts, 1986).

\subsection{Persuasion in Crawford and Sobel's model}

In this subsection, we will see that, even if the underlying persuasion game is not monotonic, sufficient conditions can be given for the existence and/or the uniqueness of a FRE. This is the aim of Seidmann and Winter (1997), who studied the transmission of certifiable information in a model that encompasses Crawford and Sobel (1982)'s one. Seidmann and Winter (1997) essentially make the assumptions detailed at the end of subsection 2.3, except for condition (C) on page 9: the sign of $D(k)=j_{2}^{*}(k)-j_{1}^{*}(k)$ is not necessarily constant. A set of types $L \subseteq K$ is certifiable if there exists a message $m$ which enables the expert to prove that his type belongs to $L$, i.e., such that $M^{-1}(m)=L$. Seidmann and Winter (1997) assume that $M^{-1}(m)$ is closed and that every singleton $\{k\}, k \in K$, is certifiable. Under the previous general assumptions and either

(a) $D(k)$ does not change sign over $K$, or

(b) $D(k)$ changes sign once over $K$, and $D(0)>0$,

they prove that there exists a fully revealing equilibrium, and that all equilibria are fully revealing.

Existence and uniqueness of the FRE are thus guaranteed in the most general model considered by Crawford and Sobel (1982), in particular in the uniform-quadratic case. Seidmann and Winter (1997) also establish the existence of a not necessarily unique FRE when $D(k)$ changes sign several times, provided that the expert's preferences satisfy a "single crossing" condition.

\section{Multistage Communication with Unverifiable Types}

In some economic interactions, the expert just prepares a technical report for the decision maker. In that case, the model of one shot, unilateral communication is fully appropriate. But in most interactive situations, the agents can talk with each other, so that multistage, bilateral communication is more natural. Even if only one of the players has private information, a conversation between the two of them enables them to generate stages of compromise, whose outcome is not determined in advance and cannot be individually controlled by any of the participants. These stages, which can be interpreted as reduced forms of negotiation, typically create uncertainty in the way information transmission will go on. Formally, the players perform jointly controlled lotteries, a tool that was first introduced in the study of repeated games with incomplete information (Aumann et al., 1968). Let us illustrate how it works on a simple example. 
Imagine that player 1 and player 2 want to choose between two outcomes $H$ and $T$ with equal probability but cannot rely on any extraneous device. They can simultaneously send one of two messages to each other, with the prescription that both of them use a fair coin. If $H$ is selected when both players send the same message and $T$ is selected otherwise, $\operatorname{Pr}(H)=\operatorname{Pr}(T)=1 / 2$ as soon as one of the players uses the prescribed strategy. Unilateral deviations are thus useless.

Combined with stages of information transmission, jointly controlled lotteries can modify the expert's incentives to reveal information, giving rise to outcomes which could never be achieved with unilateral communication, as we will see in the next subsection.

\subsection{A geometric characterization of Nash equilibria}

Let us construct, from $\Gamma$ and $p$, the communication game $\Gamma_{n}^{0}(p)$, by inserting $n$ stages of bilateral communication between the information phase and the action phase of the basic game $\Gamma(p)$. In particular, we still assume, for simplicity, that the expert has only two possible types. Let $M^{1}$ and $M^{2}$ be finite sets of messages, such that $\left|M^{i}\right| \geq 2, i=1,2$. At every stage $t=1, \ldots, n$ of the conversation phase, the expert sends a message $m_{t}^{1} \in M^{1}$ to the decision maker, and, simultaneously, the decision maker sends a message $m_{t}^{2} \in M^{2}$ to the expert. ${ }^{6}$

A history of length $t, t=0,1, \ldots, n$, is a sequence of $t$ pairs of messages, $\left(m_{1}^{1}, m_{1}^{2}, \ldots, m_{t}^{1}, m_{t}^{2}\right) \in$ $M_{t} \equiv\left(M^{1} \times M^{2}\right)^{t}$. A strategy $\sigma$ of the expert in $\Gamma_{n}^{0}(p)$ consists in a sequence of mappings $\sigma_{1}, \ldots, \sigma_{n}$, where $\sigma_{t}: K \times M_{t-1} \rightarrow \Delta\left(M^{1}\right), t=1, \ldots, n$. Similarly, a strategy $\tau$ of the decision maker is described by mappings $\tau_{1}, \ldots, \tau_{n}$, together with a function $\tau_{n+1}$, where $\tau_{t}: M_{t-1} \rightarrow \Delta\left(M^{2}\right), t=1, \ldots, n$, and $\tau_{n+1}: M_{n} \rightarrow \Delta(J)$.

Let $\mathcal{E}_{n}^{0}(p)$ be the set of Nash equilibrium payoffs of the $n$ stage bilateral communication game $\Gamma_{n}^{0}(p)$. As in the basic game $\Gamma(p)$ and the unilateral communication game $\Gamma_{S}^{0}(p)$ considered previously, the payoffs are computed at the interim stage, so that the expert's payoff is indexed by his type. Clearly, $\mathcal{E}_{S}^{0}(p) \subseteq \mathcal{E}_{n}^{0}(p) \subseteq \mathcal{E}_{n+1}^{0}(p)$ for every $n \geq 1$. Let $\mathcal{E}_{B}^{0}(p)=\bigcup_{n \geq 1} \mathcal{E}_{n}^{0}(p)$ be the set of all payoffs which can be achieved at a Nash equilibrium of some bilateral communication game, of arbitrary finite length, constructed from $\Gamma$ and $p$. The characterization of $\mathcal{E}_{B}^{0}(p)$ below is based on the results of Hart (1985) and Aumann and Hart (2003). ${ }^{7}$

As we have seen in section 2.1, provided that we slightly modify the graph of the equilibrium payoffs of the game without communication into gr $\mathcal{E}^{+}$, the condition for the expert to reveal information at an equilibrium of $\Gamma_{S}^{0}(p)$ is that his expected vector payoff be the same, whatever the message he sends. Hence, geometrically, the graph $\operatorname{gr} \mathcal{E}_{S}^{0}$ of the equilibrium payoffs achieved with a single stage of unilateral communication can be obtained by convexifying gr $\mathcal{E}^{+}$in $(\beta, p)$ while keeping constant the expert's payoff, a (see theorem 1 ). Let us

\footnotetext{
${ }^{6}$ We do not need to assume anymore that the expert has at least three messages, as we did for unilateral communication, since we can add further communication stages.

${ }^{7}$ These articles do not require that the conversation between the expert and the decision last for finitely many stages; we will comment on this later on.
} 
set $H_{1}^{0}=\operatorname{gr} \mathcal{E}_{S}^{0}=\operatorname{conv}_{a}\left(\operatorname{gr} \mathcal{E}^{+}\right) ; H_{1}^{0}$ is convex in $(\beta, p)$ when $a$ is fixed, but new points can be obtained by convexifying $H_{1}^{0}$ in $(a, \beta)$ while keeping constant the probability $p$. This is exactly the effect of a jointly controlled lottery, as seen above. The graph of the equilibrium payoffs achieved with one stage of information transmission and one jointly controlled lottery, $H_{2}^{0}$, can thus be obtained as $\operatorname{conv}_{p}\left(H_{1}^{0}\right) . H_{2}^{0}$ being not necessarily convex in $(\beta, p)$ when $a$ is fixed, one can perform another step of convexification: $H_{3}^{0}=\operatorname{conv}_{a}\left(H_{2}^{0}\right)$. A payoff $(a, \beta)$ such that $(a, \beta, p) \in H_{3}^{0}$ corresponds to an equilibrium of $\mathcal{E}_{3}^{0}(p)$ which is achieved by means of two stages of information transmission, and a compromise stage taking place between them. During that stage, both players exchange messages. The gradual convexification process just described for 3 stages can possibly go on $n=4,5, \ldots$

A subset of $\mathbb{R}^{2} \times \mathbb{R} \times[0,1]$ is diconvex if it is convex in $(\beta, p)$ when $a$ is fixed and convex in $(a, \beta)$ when $p$ is fixed. We get $\mathcal{E}_{B}^{0}(p)$ as the section in $p$ of $\operatorname{dico}\left(\operatorname{gr} \mathcal{E}^{+}\right)$, the smallest diconvex set containing gr $\mathcal{E}^{+}$. In other words:

Theorem 3 (Characterization of $\left.\mathcal{E}_{B}^{0}(p)\right)$ Let $\left.p \in\right] 0,1[$. A payoff $(a, \beta)$ is an equilibrium payoff of some bilateral communication game $\Gamma_{n}^{0}(p)$, for some length $n$, if and only if $(a, \beta, p)$ belongs to $\operatorname{dico}\left(\operatorname{gr} \mathcal{E}^{+}\right)$, the set of all points obtained by diconvexifying the set $\operatorname{gr} \mathcal{E}^{+}$:

$$
\mathcal{E}_{B}^{0}(p)=\left\{(a, \beta) \in \mathbb{R}^{2} \times \mathbb{R}:(a, \beta, p) \in \operatorname{dico}\left(\operatorname{gr} \mathcal{E}^{+}\right)\right\}
$$

See Hart (1985), Forges (1994) and Aumann and Hart (2003) for a proof. The theorem is true whatever the number of types of the expert.

A natural question is whether there are games in which some equilibrium payoffs cannot be achieved with a bounded number $n$ of communication stages, but can be reached if no deadline is imposed to the communication process. The answer is positive, as shown in Forges $(1984,1990)$ on a simple game of the same form as example 4 , with two types for the expert and five actions for the decision maker.

The equilibrium payoffs obtained by diconvexification (i.e., as in theorem 3) can also be described as starting points of particular martingales, called dimartingales (Aumann and Hart, 1986) because the $p$ - and $a$-coordinates never split simultaneously, which converge to gr $\mathcal{E}^{+}$in a finite, known in advance, number of stages. The equilibrium payoff exhibited in Forges $(1984,1990)$ is achieved through a dimartingale which converges in a finite but not bounded number of stages, which is endogenously determined by the equilibrium. Hart (1985) and Aumann and Hart (2003) even consider dimartingales which reach their nonrevealing limit in infinitely many stages. In this case, the expert never stops transmitting information, but convergence requires that he gradually reveals less and less information. According to Aumann and Hart (2003), the bilateral communication taking place before the single decision phase can last forever. We do not know of any game which would illustrate this phenomenon (see Aumann and Hart, 1986, for a geometric example, and Krishna, 2005 for a discussion of this issue). 


\subsection{Conversation in Crawford and Sobel's model}

Let us go back to the uniform-quadratic version of Crawford and Sobel (1982)'s model (see subsection 2.3), but consider now three stages of communication, consisting of two stages of information transmission from the expert to the decision maker, with a stage of bilateral communication in between. The latter stage enables the agents to generate a jointly controlled lottery as above. Krishna and Morgan (2004) show that, for intermediary values of the expert's bias (like $b=1 / 10$ ), there may exist monotonic equilibria (i.e., in which the action of the decision maker increases with the expert's type) which Pareto improve upon all equilibria of the unilateral communication game. For a high bias of the expert (say, $b=7 / 24$ ), while the only equilibrium of the unilateral communication is nonrevealing (because $b=7 / 24>1 / 4$, see subsection 2.3), there may exist a Pareto improving, non-monotonic, partially revealing equilibrium in the three stage communication game (see Krishna and Morgan, 2004, for details and statements which indicate that the previous Pareto improving equilibria are not accidental).

\section{Multistage Communication with Verifiable Types}

Consider again the persuasion game of example 4, with two equally likely states. We have already found four Nash equilibria; the partially revealing equilibrium PRE1 is the best one for the expert, with an expected payoff of 2, whatever his type. We will now show that several stages of bilateral communication allow the expert to certify his information with some delay and to increase his expected payoff up to the amount of 3 , whatever his type. This equilibrium, which is depicted on figure 9 on the next page, is achieved by means of two stages of information transmission, separated by a jointly controlled lottery (denoted as JCL) .

In the same way as the equilibrium payoffs of the multistage cheap talk games (see theorem $3)$, the expert's equilibrium payoff $(3,3)$ at $p=1 / 2$ can be constructed geometrically by diconvexifying an adapted graph of nonrevealing equilibrium payoffs. More precisely, let us consider a persuasion game $\Gamma_{n}(p)$ with the same timing as the communication game $\Gamma_{n}^{0}(p)$ of section 4.1, but in which, at every stage $t=1, \ldots, n$, the set of messages $M(k)$ that are available to the expert depends on his type $k$, exactly as in the unilateral persuasion game (see section 3.1). We still assume that the expert can remain silent, but, by increasing the number of communication stages, one can limit the number of messages. $M^{1}=M\left(k_{1}\right) \cup M\left(k_{2}\right)$ is the set of all the messages that the expert could possibly send, from the decision maker point of view. The latter can now also send a message in $M^{2},\left|M^{2}\right| \geq 2$, at every stage.

A history of length $t=0,1, \ldots, n$ in $\Gamma_{n}(p)$ can be defined as in $\Gamma_{n}^{0}(p)$ (namely, as if the types were not verifiable, since every message in $M^{1}$ is a priori conceivable, from the decision maker's point of view). In particular, $M_{t}=\left(M^{1} \times M^{2}\right)^{t}$. However, a strategy $\sigma$ of the expert in the persuasion game $\Gamma_{n}(p)$ must be defined over those messages that are really available to the expert: $\sigma$ can be described as a sequence of mappings $\sigma_{1}, \ldots, \sigma_{n}$, where $\sigma_{t}=\left(\sigma_{t}^{1}, \sigma_{t}^{2}\right)$ 


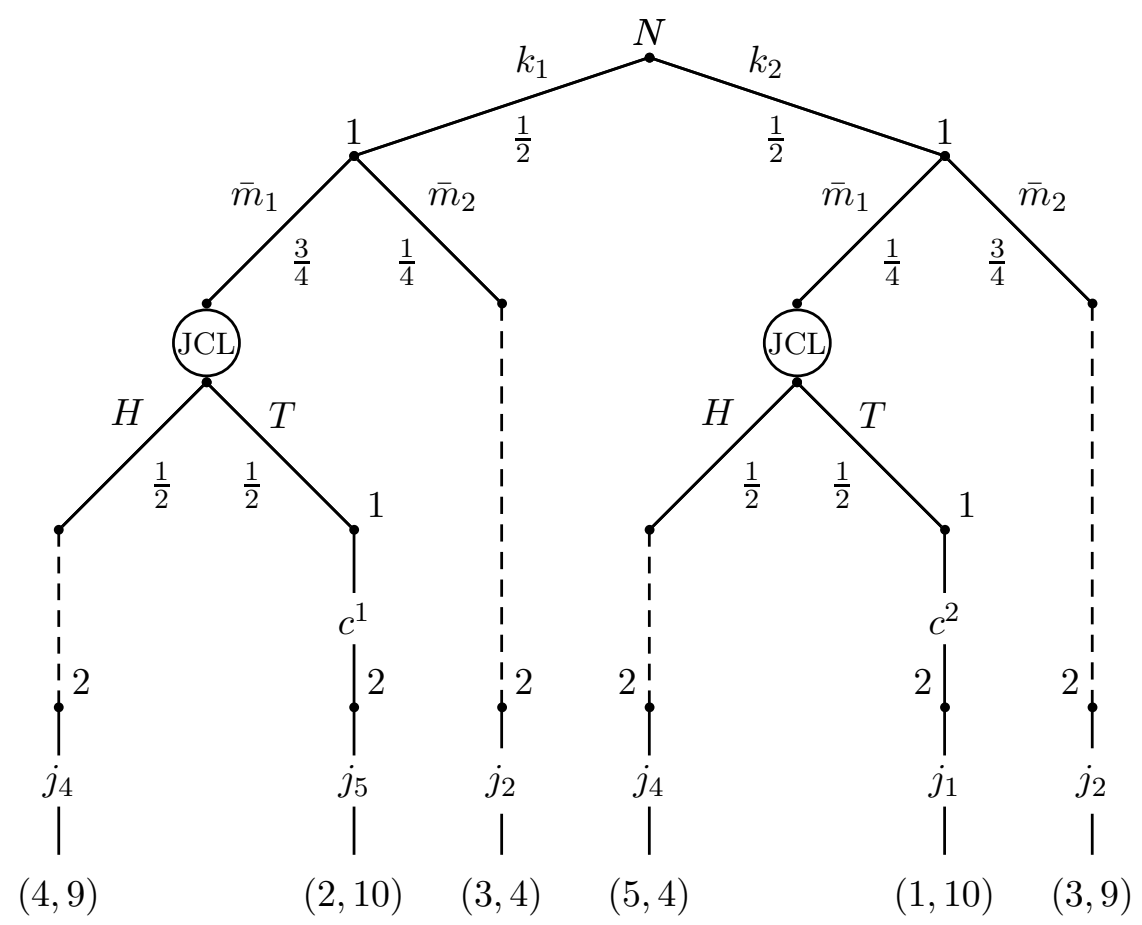

Figure 9: An equilibrium outcome of the three-stage communication game from example 4.

and $\sigma_{t}^{k}: M_{t-1} \rightarrow \Delta(M(k))$ for $k=1,2$ and $t=1, \ldots, n$. The decision maker's strategies are as in $\Gamma_{n}^{0}(p)$.

Let $\mathcal{E}_{n}(p)$ be the set of Nash equilibrium payoffs of $\Gamma_{n}(p)$. $\mathcal{E}_{S}(p) \subseteq \mathcal{E}_{n}(p) \subseteq \mathcal{E}_{n+1}(p)$ for every $n \geq 1$. Let $\mathcal{E}_{B}(p)=\bigcup_{n \geq 1} \mathcal{E}_{n}(p)$ be the set of all payoffs corresponding to some Nash equilibrium of some bilateral persuasion game constructed from $\Gamma(p)$, with an arbitrary finite number of communication stages. The next theorem gives a characterization of $\mathcal{E}_{B}(p)$ in the particular case of two types for the expert, which we have concentrated on all along the paper. The sets INTIR and gr $\mathcal{E}^{++}$are defined as in section 3.1 .

Theorem 4 (Characterization of $\mathcal{E}_{B}(p)$ ) Let $\left.p \in\right] 0,1[$. A payoff $(a, \beta)$ is an equilibrium payoff of a multistage bilateral persuasion game $\Gamma_{n}(p)$, for some length $n$, if and only if $(a, \beta, p)$ belongs to $\operatorname{dico}\left(\operatorname{gr} \mathcal{E}^{++}\right) \cap \mathrm{INTIR}$, the set of all points obtained by diconvexifying the set of all payoffs in gr $\mathcal{E}^{+}$that are interim individually rational for the expert:

$$
\mathcal{E}_{B}(p)=\left\{(a, \beta) \in \mathbb{R}^{2} \times \mathbb{R}:(a, \beta, p) \in \operatorname{dico}\left(\operatorname{gr} \mathcal{E}^{++}\right) \cap \operatorname{INTIR}\right\}
$$

See Forges and Koessler (2006) for a proof. A similar, but more intricate, characterization holds if the number of types of the expert exceeds two (see the comment below), provided that the expert can certify any subset of types $L \subseteq K$.

When the expert has only two types, the characterization of $\mathcal{E}_{B}(p)$ is a mere transposition of the one of $\mathcal{E}_{B}^{0}(p)$ (theorem 3): it suffices to replace gr $\mathcal{E}^{+}$with gr $\mathcal{E}^{++} \cap$ INTIR. One can 
indeed check that, if $|K|=2$, dico(gr $\left.\mathcal{E}^{++}\right) \cap \operatorname{INTIR}=\operatorname{dico}\left(\operatorname{gr} \mathcal{E}^{++} \cap \operatorname{INTIR}\right)$. The peculiarity of the two type case comes from the fact that as soon as the posterior probability distribution in some step $s, p_{s}$, reaches the boundary of the "simplex" (which is just an interval here), the true type of the expert is fully revealed and the game cannot evolve anymore. The individual rationality condition for the expert can be expressed as $\left(a_{s}, \beta_{s}, p_{s}\right) \in$ INTIR, one of the components of $a_{s}$ being artificially fixed at its previous value. If the expert has more than two types, a natural way to keep a similar characterization as above is to define individual rationality for the expert with respect to the posterior probability distribution in each step of the process of convexification. Such a characterization is proposed in Forges and Koessler (2006). In its general form, for $|K| \geq 2$, the result is less tractable than the corresponding one for unverifiable information (theorem 3). Indeed, due to the additional (individual rationality) constraint that is imposed all along the martingale, the latter evolves in a set which is not necessarily diconvex. Hence, there is no reason to expect the graph gr $\mathcal{E}_{B}$ of all equilibrium payoffs of the multistage persuasion game to be diconvex.

Let us illustrate theorem 4 on the equilibrium of example 4 analyzed above (figure 9 ). At $p=1 / 4$, a jointly controlled lottery followed by an information transmission stage enables us to convexify, $p$ remaining fixed, the set of all equilibrium payoffs achieved with one stage communication; we obtain the triangle $\left[j_{2}\right.$, FRE, PRE2] of figure 8 on page 12. Similarly, at $p=3 / 4$, we obtain the expert's equilibrium payoffs in the triangle $\left[j_{4}\right.$, FRE, PRE3]. A further convexification, with $a$ fixed, thus enables us to get as expert's equilibrium payoffs, for every $p \in] 1 / 4,3 / 4[$ (in particular, $p=1 / 2$ ), the intersection of the previous two triangles (in particular, $a=(3,3)$ ).

\section{Acknowledgments}

The first author is indebted to the French Ministry of Research (ACI jeunes chercheurs) for its financial support. He realized part of this work while visiting the Institute for Advanced Studies at the Hebrew University of Jerusalem (Israel).

\section{References}

Aumann, R. J. And S. Hart (1986): "Bi-Convexity and Bi-Martingales," Israel Journal of Mathematics, 54, 159-180. - (2003): "Long Cheap Talk," Econometrica, 71, 1619-1660.

Aumann, R. J., M. Maschler, and R. Stearns (1968): "Repeated Games with Incomplete Information: An Approach to the Nonzero Sum Case," Reports to the U.S. Arms Control and Disarmament Agency, ST-143, Chapter IV, pp. 117-216.

Blume, A. (1994): "Equilibrium Refinements in Sender-Receiver Games," Journal of Economic Theory, 64, 66-77. 
Crawford, V. P. And J. Sobel (1982): "Strategic Information Transmission," Econometrica, 50, 1431-1451.

Forges, F. (1984): "Note on Nash equilibria in repeated games with incomplete information," International Journal of Game Theory, 13, 179-187.

(1990): "Equilibria with Communication in a Job Market Example," Quarterly Journal of Economics, 105, 375-398.

(1994): "Non-Zero Sum Repeated Games and Information Transmission," in Essays in Game Theory: In Honor of Michael Maschler, ed. by N. Megiddo, Springer-Verlag.

Forges, F. And F. Koessler (2006): "Long Persuasion Games," THEMA Working Paper $2006-01$.

Gordon, S. (2006): "Informative Cheap Talk Equilibria as Fixed Points," mimeo.

Green, J. R. And J.-J. Laffont (1986): "Partially Verifiable Information and Mechanism Design," Review of Economic Studies, 53, 447-456.

Green, J. R. And N. Stokey (1980): "A Two-Person Game of Information Transmission," H.I.E.R. Discussion Paper No. 751, Harvard University.

Grossman, S. J. (1981): "The Informational Role of Warranties and Private Disclosure about Product Quality," Journal of Law and Economics, 24, 461-483.

Grossman, S. J. and O. D. Hart (1980): "Disclosure Laws and Takeover Bids," Journal of Finance, 35, 323-334.

HART, S. (1985): "Nonzero-Sum Two-Person Repeated Games with Incomplete Information," Mathematics of Operations Research, 10, 117-153.

Kreps, D. M. And J. Sobel (1994): "Signalling," in Handbook of Game Theory, ed. by R. J. Aumann and S. Hart, Elsevier Science B. V., vol. 2, chap. 25, 849-867.

Krishna, V. And J. Morgan (2004): "The Art of Conversation: Eliciting Information from Experts through Multi-Stage Communication," Journal of Economic Theory, 117, $147-179$.

Krishna, V. R. (2005): "Extended Conversations in Sender-Receiver Games," mimeo.

Melumad, N. D. And T. Shibano (1991): "Communication in Settings with no Transfers," Rand Journal of Economics, 22, 173-198.

Milgrom, P. (1981): "Good News and Bad News: Representation Theorems and Applications," Bell Journal of Economics, 12, 380-391. 
Milgrom, P. And J. Roberts (1986): "Relying on the Information of Interested Parties," Rand Journal of Economics, 17, 18-32.

Renault, J. (2000): "On two-player repeated games with lack of information on one side and state-independent signalling," Mathematics of Operations Research, 25, 552-572.

Seidmann, D. J. and E. Winter (1997): "Strategic Information Transmission with Verifiable Messages," Econometrica, 65, 163-169.

Simon, R., S. Spiez, And H. Torunczyk (1995): "The existence of equilibria in certain games, separation for families of convex functions and a theorem of Borsuk-Ulam type," Israel Journal of Mathematics, 92, 1-21.

SorIN, S. (1983): "Some results on the existence of Nash equilibria for non zero-sum games with incomplete information," International Journal of Game Theory, 12, 193-205.

Watson, J. (1996): "Information Transmission when the Informed Party is Confused," Games and Economic Behavior, 12, 143-161. 